\title{
Competency of Clove and Cinnamon Essential Oil Fumigation against Toxigenic and Atoxigenic Aspergillus flavus Isolates
}

\author{
Sansern Rangsuwan ${ }^{1}$ iD, Chainarong Rattanakreetakul ${ }^{1,2 *}$ \\ and Ratiya Pongpisutta ${ }^{1,2}$ (D) \\ ${ }^{1}$ Department of Plant Pathology, Faculty of Agriculture at Kamphaeng Saen, Kasetsart University, Nakhon \\ Pathom 73140, Thailand. \\ ${ }^{2}$ Postharvest Technology Innovation Center, Ministry of Higher Education, Science, Research and Innovation, \\ Bangkok 10400, Thailand.
}

\begin{abstract}
Aspergillus flavus is a frequent contaminant of maize grain. We isolated this fungus, determined the colony morphology and species (by internal transcribed spacer sequencing) and measured the aflatoxin content. The selected $A$. flavus fungi were placed into two groups, toxigenic and atoxigenic; both appeared similar morphologically, except that the atoxigenic group lacked sclerotia. An essential oil fumigation test with clove and cinnamon oils as antifungal products was performed on fungal conidial discs and fungal colonies in Petri plates. Cinnamon oil at 2.5 to $5.0 \mu \mathrm{L} / \mathrm{plate}$ markedly inhibited the mycelial growth from conidial discs of both strains, whereas clove oil showed less activity. The oils had different effects on fungal mycelia. The higher clove fumigation doses of 10.0 to $20.0 \mu \mathrm{L} /$ plate controlled fungal growth, while cinnamon oil caused less inhibition. Compared with atoxigenic groups, toxigenic $A$. flavus responded stably. Within abnormal $A$. flavus hyphae, the essential oils degenerated the hyphal morphology, resulting in exfoliated flakes and shrinkage, which were related to fungal membrane injury and collapse of vacuoles and phialide. The treatments, especially those with cinnamon oil, increased the electroconductivity, which suggested a weak mycelium membrane structure. Moreover, the treatments with essential oils reduced the ergosterol content in mycelia and the aflatoxin accumulation in the culture broth. The fumigations with clove and cinnamon oils inhibited the development of both conidia and colonies of $A$. flavus in dose-dependent manners.
\end{abstract}

Keywords: Natural product, Antifungal, Aflatoxin, Storage fungi

*Correspondence: chainarong.r@ku.th; +6692-464-4524

(Received: June 15, 2021; accepted: June 30, 2021)

Citation: Rangsuwan S, Rattanakreetakul C, Pongpisutta R. Competency of Clove and Cinnamon Essential Oil Fumigation against Toxigenic and Atoxigenic Aspergillus flavus Isolates. J Pure Appl Microbiol. 2021;15(3):1325-1337. doi: 10.22207/JPAM.15.3.23

(C) The Author(s) 2021. Open Access. This article is distributed under the terms of the Creative Commons Attribution 4.0 International License which permits unrestricted use, sharing, distribution, and reproduction in any medium, provided you give appropriate credit to the original author(s) and the source, provide a link to the Creative Commons license, and indicate if changes were made. 


\section{INTRODUCTION}

Maize (Zea mays L.) is an important food crop produced by smallholder farmers. The crop provides household food security through income generation. Harvested grain may contain storage fungi that cause spoilage during storage. The grain losses caused by storage fungi pose a significant constraint on household food security. Most of the postharvest processing in the food and feed industry focuses on the contaminants of storage fungi such as Aspergillus sp., Penicillium sp. and Fusarium sp. More than 300 Aspergillus spp. were reported to have different chemodiversity among the species ${ }^{1,2}$. $A$. flavus is one of the active mycotoxin-producing specie. Fungi produce aflatoxin $\mathrm{B} 1$ and aflatoxin $B 2^{3-6}$. They can reduce the quality of human and animal health? ${ }^{7}$. Fungi affect stored grain quality due to storage conditions in the storage location, e.g., a silo. Various control measures are practiced to prevent those mycotoxin problems, such as cleaning, sorting of clean grain, and decreasing the grain moisture content. Controlling the moisture content helps to reduce the growth of spoilage fungi such as Aspergillus sp., Penicillium sp., Fusarium sp., and other postharvest fungi ${ }^{8}$. Fresh harvested grains with a moisture content of $23 \%$ were dried until they reached a lower limit of $13 \%$ before storage in a cabin. The grain was collected in a woven polypropylene bag or covered with a woven polypropylene sheath, resulting in an increase in moisture content under the cover polypropylene sheath. High humidity and temperature in the heaped maize provided favorable conditions for fungal growth. Delays in the dry grain process caused an increase in fungal contamination ${ }^{9}$.

Natural products from essential oils do not pollute the environment, and consequently, they offer more public usage acceptance. One herbal activity is fungicidal activity ${ }^{10-14}$. Many works also report activities against bacteria ${ }^{15,16}$ and insects $^{17,18}$. Essential oils such as those of clove, mountain thyme, and anise showed excellent inhibition against storage fungi ${ }^{19,20}$, reducing fungal growth and aflatoxin production. Nevertheless, most essential oil studies have focused on their activities on target cells with direct contact assays. In contrast, the application of essential oils in a closed system, such as a silo, or storage under a propylene sheath needs to be considered for their activities using fumigation or vapor approaches. Applications of essential oils may be derived from their activities.

In general, essential oil research has applied essential oils as liquid sprays to crop products. The application stands for the ideal of essential oils fumigation against storage fungi. The knowledge can be applied to the further use of essential oils on grain, improving the stored grain quality by preventing mold growth and aflatoxin production. This study aimed to evaluate the fumigation of clove and cinnamon oil to control $A$. flavus and the fungal response after the essential oils treated.

\section{MATERIALS AND METHODS}

Isolation of Aspergillus spp. from maize grain and their potential for aflatoxin production

Fresh harvested maize grain was collected from a cultivation area in Nakhon Pathom Province, Thailand. After harvest, one kilogram of grain was piled on a clean tray, flattened, and spread to cover the tray. After dividing the sample into four parts, the sampled grains were collected from two parts. Then, the sample was remixed, and the process was repeated until the mass of the grain was reduced to one hundred grams. One hundred maize seeds (10 seeds per plate) were plated on malt salt agar (MSA) (2 g of malt extract, $7.5 \mathrm{~g}$ of sodium chloride, and $1.5 \mathrm{~g}$ of agar in 100 $\mathrm{mL}$ ). Petri plates were incubated at $25^{\circ} \mathrm{C}$ under a 12/12 hr light/dark cycle for 7 days. Each grain was observed under a stereo microscope, and an Aspergillus colony was isolated from each infected seed. The morphologies of the Aspergillus sp. colonies were observed on Czapek's agar following Raper and Fennel's (1977) ${ }^{21}$ work. After observing the colony characteristics and the vesicle type under a compound microscope, A. flavus was cultured in potato dextrose agar (PDA) and kept for further study.

A. flavus was screened for aflatoxin production under axenic liquid culture. A spore suspension (one hundred microliters of $1.0 \times 10^{6}$ conidia/mL in $0.01 \%$ Tween 20 ) was inoculated into $100 \mathrm{~mL}$ of yeast extract sucrose (YES) broth $(4 \mathrm{~g}$ of yeast extract, $20 \mathrm{~g}$ of sucrose, $1 \mathrm{~g}$ of potassium 
dihydrogen phosphate, $0.5 \mathrm{~g}$ of magnesium sulfate, and $1,000 \mathrm{~mL}$ of distilled water). After five days at $28^{\circ} \mathrm{C}$, the fungal culture medium was used to determine the aflatoxin content in broth by an AgraQuant ${ }^{\circledR}$ Total Aflatoxin ELISA Test (Romer, Getzerdorf, Austria).

The sequences of the internal transcribed spacer (ITS) region confirmed the species $A$. flavus. Fungal genomic DNA was extracted by a DNA secure Plant Kit (Tiangen, Beijing, China). The ITS region was amplified with the primers ITS1 (5'-TCC GTA GGT GAA CCT GCG G-3') and ITS4 (5'-TCC TCC GCT TAT TGA TAT GC-3') (Sigma-Aldrich, Merck, Darmstadt, Germany). PCR amplification was carried out in $20 \mu \mathrm{L}$ reactions containing $2 \mu \mathrm{L}$ of template DNA (30 ng), $2 \mu \mathrm{L}(2 \mathrm{mM})$ of each primer, $2 \mu \mathrm{L}$ of $10 \mathrm{X}$ PCR buffer (with $\mathrm{KCl}$ ), $2 \mu \mathrm{L}$ of $2 \mathrm{mM}$ $\mathrm{MgCl} 2,2 \mu \mathrm{L}$ of $2 \mathrm{mM}$ dNTPs $(2.0 \mathrm{mmol} / \mathrm{L})$, and 0.5 $\mu \mathrm{L}$ of Taq polymerase $(5 \mathrm{U} / \mu \mathrm{L})$ and adjusted to $20 \mu \mathrm{L}$ with ddH2O. PCRs were performed on a PCR cycler (SensoQuest, Gottingen, Germany). The thermal cycling program was predenaturation at $94^{\circ} \mathrm{C}$ for 2 min, followed by 30 cycles of denaturation at $93^{\circ} \mathrm{C}$ for $30 \mathrm{~s}$, annealing at $56.5^{\circ} \mathrm{C}$ for $30 \mathrm{~s}$, and extension at $72^{\circ} \mathrm{C}$ for $1 \mathrm{~min}$, with a final elongation step of $72^{\circ} \mathrm{C}$ for $10 \mathrm{~min}$. PCR products were sequenced by First BASE Laboratories (Selangor, Malaysia). The sequence was analyzed, and similar sequences were sought using the Basic Local Alignment Search Tool (BLAST) at the National Center for Biotechnology Information (NCBI).

Antifungal efficiencies of clove and cinnamon oils in fumigation assays and contact assays

The growth of both strains of $A$. flavus was tested in two fungal developmental stages: as conidial discs, conidia were spread on the surface of agar medium, and as fungal colonies on PDA. They were treated with clove oil (CAS number 8000-34-8) and cinnamon oil (CAS number 8464998-9) from Sigma-Aldrich Pte., Ltd., in a fumigation assay in Petri plates. In the conidial disc assays, clove and cinnamon oils were added at fumigation rates of $1.25,2.5$ and $5.0 \mu \mathrm{L} /$ plate. The essentials oils were mixed with acetone to a desired volume, and twenty microliters of each was dropped on the filter paper. The essential oils were set above the tested fungi in Petri plates. One square centimeter of filter paper was fixed on a Petri plate lid. After dropping essential oil on the filter paper, the Petri plate was wrapped with parafilm to avoid essential oil vapor leaks. For fungal colony tests, a two-day-old $A$. flavus fungal culture was prepared on PDA. Clove and cinnamon oils with fumigation rates of 5.0, 10.0 and $20.0 \mu \mathrm{L}$ /plate were used. After 2, 4, 6 and 8 days of incubation at room temperature, colony growth was measured. Mycelial growth was used to calculate the percent fungal inhibition. Abnormal colony characteristics were observed under a stereo microscope and a compound microscope after five days of incubation at room temperature. The fungal morphology was observed after essential oils were added to characteristic $A$. flavus colonies, and the fungal cells were monitored with stereo and compound microscope.

The fungal mycelium toxicity test was performed via direct mycelium contact. The essential oils were dissolved in $0.001 \%$ Tween 80 before they were mixed into culture medium to final concentrations of 100,200 , and $400 \mu \mathrm{L} / \mathrm{L}$. Conidial discs of $A$. flavus were transferred to the middle of Petri plate and incubated at room temperature. Fungal growth was measured every 2 days for 8 days. The percentage of fungal mycelium growth inhibition was calculated as $((A-B) / A))$ $\mathrm{x} 100$, where

$A=$ fungal mycelium growth measured on control agar medium and

$B=$ fungal mycelium growth measured on treated agar medium.

Assessment of the effect of clove and cinnamon oil on the A. flavus mycelium response Fungal fresh weight

The fungal fresh weight was determined after essential oil treatment. One hundred microliters of an $A$. flavus spore suspension at $10^{5}$ spores $/ \mathrm{mL}$ was inoculated in a $1,000 \mathrm{~mL}$ laboratory bottle with $50 \mathrm{~mL}$ of YES broth. The tested essential oils were amended with $0.001 \%$ Tween 80 solutions before they were dropped into YES medium at 100, 200, and $400 \mu \mathrm{L} / \mathrm{L}$ to determine the effect of concentration. The flasks were kept still at room temperature. After 10 days, the fungal biomass was filtered with Whatman filter paper No. 1. The supernatant was rinsed and collected to analyze the electroconductivity and aflatoxin content. After inverting the tube for 10 $\mathrm{min}$, the excess medium was eliminated. The fresh 
fungal weight was determined for each treatment and used to determine the ergosterol content.

\section{Fungal ergosterol content}

Ergosterol extraction was carried out with some modifications according to YamamotoRibeito et $\mathrm{al}^{22}$ and Ferreira et $\mathrm{al}^{23}$. Fungal mycelia were used to analyze the ergosterol content. Fifty milligrams of tested fungal mycelia was transferred to a test tube, and $5 \mathrm{~mL}$ of $25 \%$ alcoholic potassium hydroxide $(2.0 \mathrm{~g}$ of potassium hydroxide, 20 $\mathrm{mL}$ of methanol, $5 \mathrm{~mL}$ of ethanol) was added. The mixture was vortexed for $2 \mathrm{~min}$ followed by incubation at $70^{\circ} \mathrm{C}$ for $40 \mathrm{~min}$. Sterols were extracted from each sample by adding a mixture of $2 \mathrm{~mL}$ of sterile distilled water and $5 \mathrm{~mL}$ of $\mathrm{n}$-hexane. The mixture was sufficiently mixed by vortexing for 2 min and allowed to separate into layers for one hour at room temperature. The n-hexane layer was separated into a new test tube. This extraction was repeated twice. The $\mathrm{n}$-hexane fractions were mixed and taken to dryness with a pure nitrogen flow. The samples were stored $\left(-20^{\circ} \mathrm{C}\right.$ in darkness) until HPLC analysis. Each sample was redissolved in 1,000 $\mu \mathrm{L}$ of methanol. For HPLC analysis, an SPD 20A UV/Vis detector (Shimadzu, Kyoto, Japan) with an isocratic pump with methanol (100 v/v, HPLC-grade) was used with twenty microliters of the prepared sample. An ODS-3 column (GL Sciences, Tokyo, Japan; $5 \mu \mathrm{m}, 250 \times 4.6 \mathrm{~mm}$ ) was used. The mobile phase was applied at $1.0 \mathrm{~mL} /$ $\mathrm{min}$. The UV/Vis detection wavelength was set at $282 \mathrm{~nm}$ for determining ergosterol. Each sample was injected twice in the HPLC. The sample peak areas were compared with the standard peak areas of ergosterol determined between 20 and $100 \mu \mathrm{g} /$ $\mathrm{mL}$.

Fungal electroconductivity and aflatoxin content Fungal cell-free medium was used to analyze the electroconductivity (EC) and aflatoxin content. The medium from each sample was used to measure the EC value with a LAQUAtwin EC-22 (Horiba, Kyoto, Japan). The broth medium was diluted with $100 \%$ methanol until the sample was $70 \%$ methanol. Then, samples were analyzed by the AgraQuant ${ }^{\circledR}$ Total Aflatoxin ELISA Test (Romer, Getzerdorf, Austria) and an ELISA reader at a wavelength $450 \mathrm{~nm}$ (Tecan, Hombrechtikon, Switzerland).

\section{RESULTS}

Isolation of Aspergillus sp. from stored maize grain

Aspergillus spp. were isolated from maize grain in 2019 from Nakhon Pathom, Thailand. Aspergillus spp. were found to be a major storage fungus after 7 days of plating maize grains on MSA medium. Two characteristic Aspergillus sp. colonies were isolated: one with a thick, dark

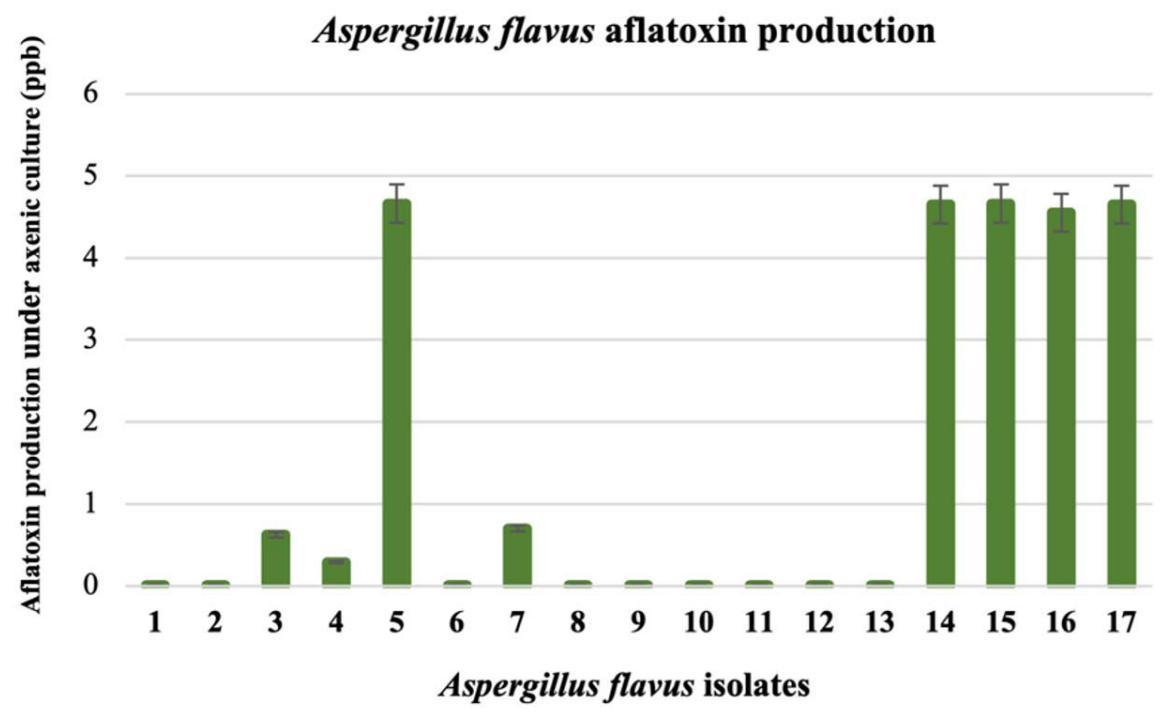

Fig. 1. Differences in aflatoxin production by seventeen Aspergillus flavus isolates in YES broth after 5 days; aflatoxin was determined by the AgraQuant ${ }^{\circledR}$ Total Aflatoxin ELISA Test.

Journal of Pure and Applied Microbiology 
green conidial head, and the other with a light brown colony with sclerotia and fewer conidial heads. Seventeen isolates of Aspergillus spp. were obtained, and they were characterized on Czapek's agar. The microscopic results showed a fungal vesicle with a globose/subglobose shape with uniseriate or biseriate phialide. The conidial head color on Czapek's agar was yellow green in young culture and turned dark green at the conidial head. The conidiophores of $A$. flavus are hyaline with a thick-walled branch arising perpendicularly from the foot cell.

All seventeen A. flavus isolates were analyzed for their aflatoxin-producing ability under YES axenic culture. The aflatoxin content from five-day-old culture broth was determined with an AgraQuant ${ }^{\circledR}$ Total Aflatoxin ELISA Test (Fig. 1). According to the aflatoxin contents, we grouped $A$. flavus into two groups. One group included isolates $1,2,3,4,6,7,8,9,10,11,12$, and 13 , which
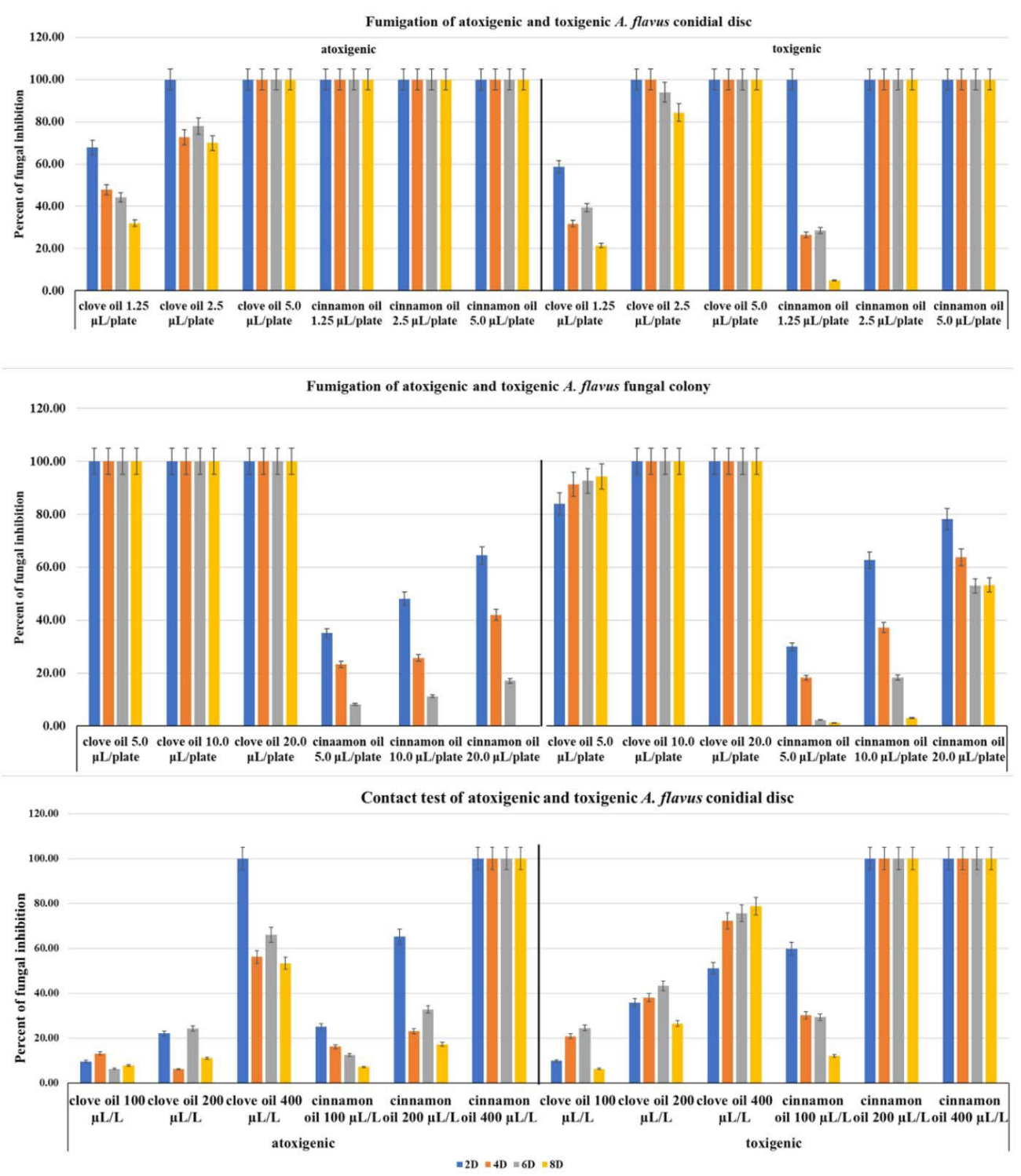

Fig. 2. Extreme control of atoxigenic and toxigenic $A$. flavus with clove and cinnamon oils during 2 to 8 days (upper) of fumigation of $A$. flavus conidial discs at $1.2-5.0 \mu \mathrm{L} /$ plate, (middle) of fumigation of $A$. flavus fungal colonies at $5.0-20.0 \mu \mathrm{L} /$ plate, and (lower) direct contact of $A$. flavus conidial discs at $100-400 \mu \mathrm{L} / \mathrm{L}$. 
produced less aflatoxin $(0.00-0.69 \mathrm{ppb})$. This $A$. flavus group was related to a fungal colony with an abundant yellow-green conidial head covering the fungal colonies. The other group with sclerotia produced more aflatoxin (reaching as high as 4.56 to $4.66 \mathrm{ppb}$ ) and included isolates $5,14,15,16$, and 17.
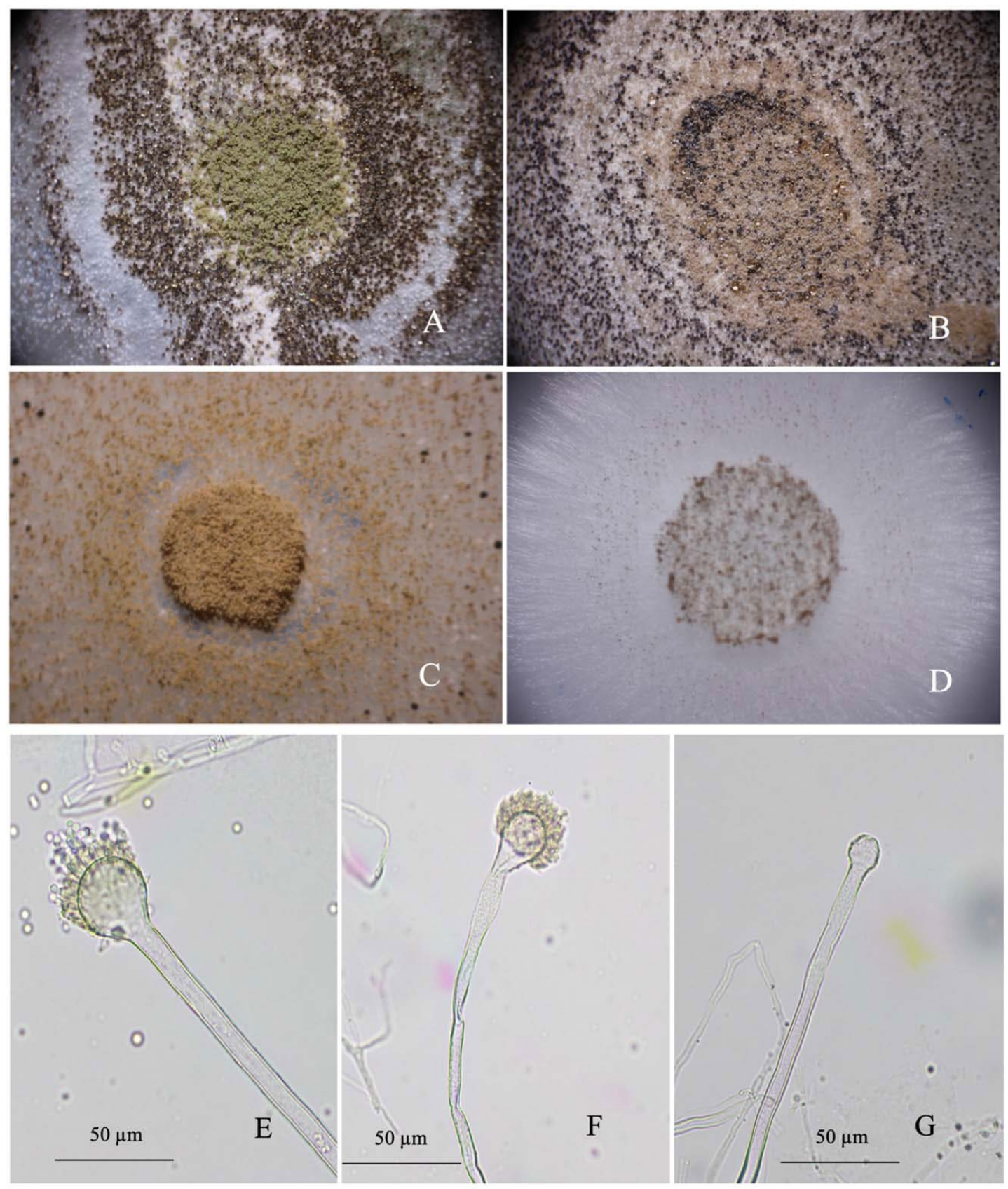

Fig. 3. Stereomicrographs of differential responses of toxigenic $A$. flavus to essential oil treatment observed at 400x: control (A), contact test with a conidial disc (B), fumigation test with a conidial disc (C), and fumigation with fungal mycelia (D); Photomicrographs of the response of Aspergillus flavus mycelia to fumigation with essential oils under compound microscope (E) untreated, $(F)$ a wrinkle on the surface of a conidiophore after treatment with clove oil, and (G) size reduction and non-phialide of a vesicle after treatment with cinnamon oil. 
Atoxigenic $A$. flavus isolate 8 and toxigenic A. flavus isolate 17 were chosen for further study. Specific characteristics of toxigenic $A$. flavus are its abundant sclerotia with a diameter of less than 400 $\mu \mathrm{m}$ and smaller conidial head. This fungal group can be classified into the $S$ strain group of $A$. flavus (group IA) ${ }^{2,3}$. After the nucleotide sequences of the ITS region of both fungal isolates were determined with ITS1/ITS4 primers, they were compared to sequences on the NCBI database (www.ncbi.nlm. nih.gov search on June 14, 2021). Both fungal ITS sequences were highly similar to those of $A$. flavus with internal transcribed spacer 1 , partial sequence; $5.8 \mathrm{~S}$ ribosomal RNA gene and internal transcribed spacer 2 . The sequence of $A$. flavus isolate 8 has been deposited in the GenBank database under accession number MZ379467 and MZ379482 for A. flavus isolate 17. Even though the two $A$. flavus strains showed similar molecular characteristics, they were diverse in aflatoxin production under broth culture. This finding for atoxigenic and toxigenic strains of $A$. flavus was described earlier by Cotty².

In situ testing of essential oil antifungal activity against $A$. flavus

Fumigation with clove and cinnamon oil resulted in different levels of fungal inhibition for conidial discs and the fungal colonies of both A. flavus strains. Cinnamon oil treatment at concentrations of 2.5 and $5.0 \mu \mathrm{L} /$ plate exhibited inhibition of all tested $A$. flavus strains that was better than that of clove oil treatment (Fig. 2 upper). Atoxigenic $A$. flavus showed growth sensitivity to cinnamon oil treatment, whereas the toxigenic strain slightly tolerated cinnamon treatment. In the fungal mycelia tested, $A$. flavus fungal colonies were fumigated with clove and cinnamon oils at 10.0 to $20.0 \mu \mathrm{L} /$ plate (Fig. 2 middle). A. flavus fungal colony growth was highly inhibited by clove oil fumigation. In contrast, fumigation with cinnamon oil showed less fungal inhibition. This means that clove oil presents greater inhibition of the fungal colonies for both strains of $A$. flavus than cinnamon oil.

In contact assays, cinnamon oil showed higher mycelium inhibition against $A$. flavus conidial discs than clove oil did (Fig. 2 lower). The cinnamon oil at a concentration of $400 \mu \mathrm{L} / \mathrm{L}$ showed total fungal growth inhibition in both tested fungi, while clove oil demonstrated less

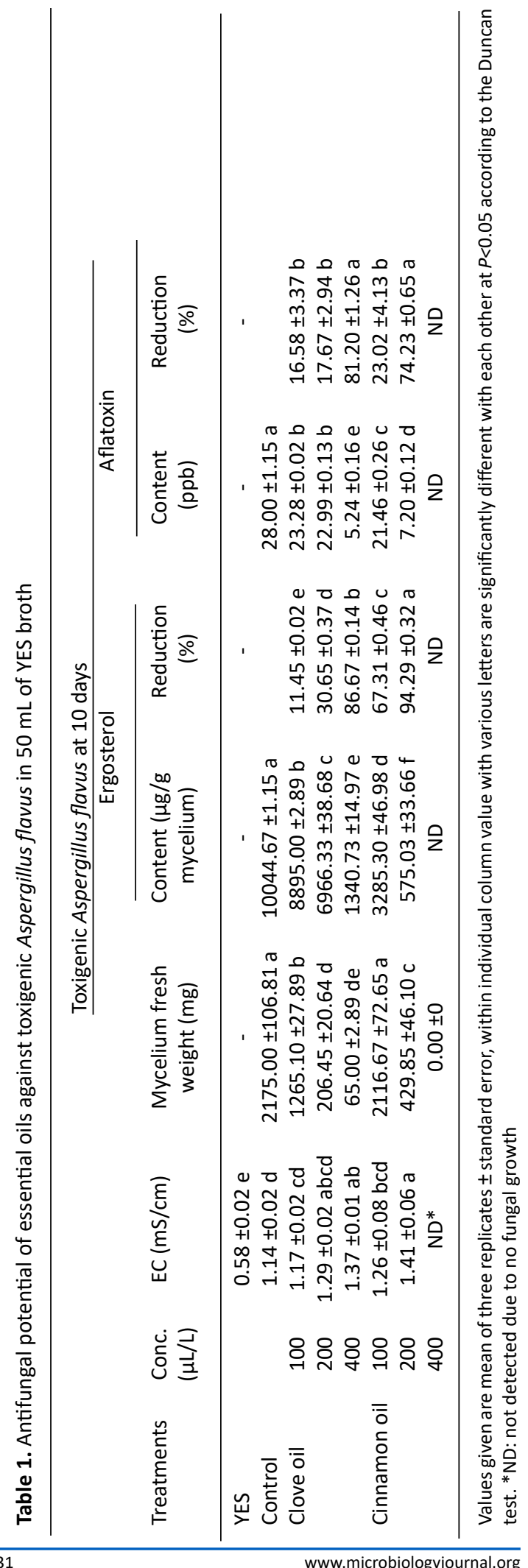

Journal of Pure and Applied Microbiology 
efficiency in controlling $A$. flavus mycelia, at $61.2-83.4 \%$ of the cinnamon oil value. Cinnamon oil showed aggressive control of both $A$. flavus strains when the inoculum source was a conidial disc. The toxigenic $A$. flavus strains were slightly more sensitive to cinnamon oil than the atoxigenic strain. Application methods such as fumigation and direct contact with essential oils show inhibition. The responses of test organisms depend on the concentrations or evaporation rates of essential oils.

Toxigenic A. flavus colonies responded to treatment with essential oils (Fig. 3 ). The conidial morphology and colonies of $A$. flavus after treatment with clove and cinnamon oils appeared abnormal. The abnormality level of the colony varied with the essential oil fumigation dose and the type of fungal inoculum. In the fumigation assays of the A. flavus colonies (Fig. $3 C$ and D), the fungal colonies exhibited conidial head color changes from green to brown (Fig. 3C), fewer conidial heads or no conidial head formation, and fewer sclerotia (Fig. 3D) after essential oil treatment. For contact assays (Fig. 3B) the fungal colony shrank, and the conidial head size and the sclerotium numbers on the agar surface decreased. These $A$. flavus phenomena resulting from essential oil treatment may be reflected in abnormalities in fungal mycelia and the fungal conidial head. At high doses for both application methods, only mycelia were produced. The fungal mycelium production was inhibited, and sclerotia were absent in both fumigation and contact assays.

The structure of the conidial head, especially the vesicle and conidiophores, was compared between treated and untreated samples. The fungal morphological characteristics were abnormal in the $A$. flavus treated with clove and cinnamon oils. In Fig. $3 E$ to $G$, the fungal vesicle and phialide are compared. In the untreated group, the vesicle showed typical fungal characteristics with complete vesicles, healthy phialide, and conidiophores near the vesicle with a coarse surface (Fig. 3E). Mycelia were straight and firm. In the treated fungal mycelia (Fig. 3F and $\mathrm{G}$ ), a higher dose of essential oil reduced the phialide, changed the vesicle shape, and withered the conidiophore. The fungal vesicle was reduced in size and sometimes exhibited a wrinkle on the surface or an abnormal shape. Phialide exhibited a shorter length than usual, or no phialide was produced. The fungal mycelia could appear as a malformation and slightly showed a withered character. This observation indicates that when treated with essential oils, the fungal mycelia were disturbed.

Effects of essential oils on the biochemical responses of $A$. flavus.

The toxigenic $A$. flavus had different responses to the direct contact with essential oils in culture medium. Clove oil and cinnamon oil at 100-400 $\mu \mathrm{L} / \mathrm{L}$ reduced fungal mycelial growth, as evidenced by fresh weight data and electrical conductivity data (Table 1). Essential oils at concentrations of 100 to $400 \mu \mathrm{L} / \mathrm{L}$ reduced the fungal mycelium fresh weight. Especially, in the cinnamon oil treatments at $400 \mu \mathrm{L} / \mathrm{L}$, the fresh fungal weight disappeared. This result indicates that the essential oils at some concentrations reduced the fungal mycelium fresh weight.

In parallel to the essential oil test, the toxigenic $A$. flavus culture broth was determined to have electrical leakage assessed as the electrical conductivity value. A lower concentration of clove and cinnamon oil, $100 \mu \mathrm{L} / \mathrm{L}$, showed no significant effect on the electrical conductivity value compared with that of untreated culture broth. However, for clove and cinnamon oils at $400 \mu \mathrm{L} / \mathrm{L}$, large increases in the electrical conductivity of 1.37 and $1.41 \mathrm{~ms} / \mathrm{cm}$ were observed, respectively (Table 1). The lower essential oil concentration showed a lower EC value, which means less mycelium leakage occurred due to the essential oils. This result revealed the efficiency with which essential oils affected the fungal growth and the disruption of fungal mycelia. Cinnamon oil inhibited the toxigenic $A$. flavus mycelia very well and better than clove oil (Table 1).

Some substances produced by toxigenic A. flavus, such as ergosterol and aflatoxin, were determined (Table 1). The data show that clove oil at $400 \mu \mathrm{L} / \mathrm{L}$ reduced the ergosterol content to $86.67 \%$. Treatment with $200 \mu \mathrm{L} / \mathrm{L}$ cinnamon oil reduced the ergosterol content to $94.29 \%$. Cinnamon showed an excellent profile for ergosterol reduction. The reduction in ergosterol content can support the hypothesis that both essential oils affect the fungal cell wall, as seen in the decrease in fresh weight and the increase in electroconductivity. 
The efficacy of essential oils on the aflatoxin production of toxigenic $A$. flavus was examined. Essential oils at higher concentrations suppressed aflatoxin production. At lower essential oil concentrations, more aflatoxin was observed; however, it was retained at levels similar to those of the control. At higher concentrations, such 400 $\mu \mathrm{L} / \mathrm{L}$ for clove oil and $200 \mu \mathrm{L} / \mathrm{L}$ for cinnamon oil, the aflatoxin content decreased to 66.32 and $68.52 \%$, respectively.

Clove and cinnamon oils inhibit toxigenic A. flavus, a cause of aflatoxin contamination in stored grain. Essential oils can directly affect storage fungi, but their efficacies depend on the fungal forms under storage conditions. Young mycelia showed higher sensitivity to both fumigation and contact with essential oils, while old mycelia resisted the essential oils. Additionally, the essential oils inhibited fungal mycelium growth and caused cell leakage, which reduced the fungal fresh weight, increased the electroconductivity, and reduced fungal ergosterol and aflatoxin contents.

\section{DISCUSSION}

Maize grain samples from Nakhon Pathom, Thailand, were sampled in 2019. After being plated on MSA, Aspergillus spp. were found to be the major component of the storage mold. Fungi have been reported worldwide to have a significant impact on stored grain. The colony and morphological characteristics of selected Aspergillus spp. on Czapek's agar were characteristic, as described by Raper and Fennell ${ }^{21}$. Most of the fungi were $A$. flavus. Aspergillus section Flavi is a dangerous fungal group that produces mycotoxin and impacts various agricultural products. The ITS sequence of ribosomal RNA in White et $\mathrm{al}^{24}$ supports the identification and classification of fungal groups. A database of ITS regions from various species of fungi is used for standard barcoding to identify genetic diversity among fungal species ${ }^{25}$. Riaz et $\mathrm{al}^{26}$ also successfully used ITS-1F and ITS-4R primers for identification of $A$. flavus species. In our study, all mentions of $A$. flavus referred to the morphological and molecular data of $A$. flavus.

All $A$. flavus samples from our study were separated into groups that produced or did not produce aflatoxin. The sclerotium and aflatoxin production of $A$. flavus were highly related. Cotty ${ }^{3,27}$ described the aflatoxin potential of Aspergillus section Flavi with $\mathrm{S}$ strain and $\mathrm{L}$ strain criteria. Additionally, Saito and Tsuruta ${ }^{28}$ described $A$. flavus species from Thailand using sclerotium production and data on the production of different mycotoxins, such as AFB1, AFB2, $A F G 1$, and AFG2. Additionally, Benito et a ${ }^{29}$ also described the $A$. flavus group $L$ strains (average sclerotium diameter $>400 \mu \mathrm{m}$ ), $\mathrm{S}$ strains (with an average sclerotium diameter $<400 \mu \mathrm{m}$ ), and NS (no sclerotia) strains. The $\mathrm{S}$ strains of Aspergillus spp. produce higher aflatoxin contents than other strains. Some studies, such as Goto et $\mathrm{al}^{30}$, detected black and pear-shaped sclerotia identified as $A$. tamarii, while $A$. flavus showed brown sclerotia. Furthermore, metabolic products of toxigenic A. flavus, such as 9-octadecenoid, were found. This substance was related to the biosynthesis of aflatoxin, and it can be found in only toxigenic A. flavus isolates ${ }^{31}$. The distribution of aflatoxinproducing fungi isolates is of concern. Mamo et $\mathrm{al}^{32}$ reported that $61 \%$ of toxigenic $A$. flavus in China had sclerotia. However, in Argentina, five to twenty-five percent of $A$. flavus isolates were found to be toxigenic strains ${ }^{29}$. In our observation, 20 to $40 \%$ of the $A$. flavus isolates were toxigenic with small sclerotia and high aflatoxin production. These fungi can occur elsewhere and can be found together with $A$. flavus without sclerotia that do not produce aflatoxin.

Essential oils with vapor activity were used to inhibit fungal mycelium growth to substantially decrease pesticide usage. Plant essential oils are known for intense antimicrobial activity $^{33}$ and they also show high potential when applied as vapor to control fungi ${ }^{34}$. The essential oils cymbopogon oil ${ }^{10,35}$, curcuma oil ${ }^{13,14}$, cinnamon oil $^{36,37}$, and clove oil ${ }^{38}$ are active against fungi, especially $A$. flavus. Hua et $\mathrm{al}^{39}$ used natural cinnamaldehyde, cinnamon oil, and synthetic cinnamaldehyde against $A$. ochraceus, and $\mathrm{Li}$ et $\mathrm{a}^{40}$ showed that Litsea cubeba oil vapor at a concentration of $1.5 \mu \mathrm{L} / \mathrm{mL}$ absolutely stopped A. flavus mycelium growth. In addition to other essential oils, cinnamon and clove oils were the focus of our previous study on their antimicrobial activities, especially against fruit postharvest fungi such as Colletotrichum gloeosporioides and Lasiodiplodia spp..$^{41,42}$ Additionally, volatiles 
from Alcaligenes faecalis completely inhibited contaminating Aspergillus sp. and reduced aflatoxin in maize, groundnut rice, and bean ${ }^{43}$.

Fumigation and contact assays were performed to estimate the essential oil fungal inhibition activity. Both application methods show promise for controlling the tested fungi. In this study, fumigation with clove oil inhibited conidial disc growth of both $A$. flavus strains with a minimal inhibition concentration (MIC) of 5 $\mu \mathrm{L} /$ plate after 8 days; the MIC for fumigation of the atoxigenic strain with cinnamon was lower than $1.25 \mu \mathrm{L} /$ plate, and the MIC for the toxigenic strain was approximately $2.5 \mu \mathrm{L} /$ plate. The MIC for direct contact with atoxigenic isolates was $400 \mu \mathrm{L} / \mathrm{L}$, while for toxigenic isolates, the MIC was $200 \mu \mathrm{L} / \mathrm{L}$. The MICs in each study indicated different fungal inhibition outcomes, as Carmo et $\mathrm{a}^{36}$ reported a similar result in contact assays against Aspergillus spp. with an MIC50 value of 40 $\mu \mathrm{L} / \mathrm{mL}$ and an MIC90 value of $80 \mu \mathrm{L} / \mathrm{mL}$. However, Xing et $\mathrm{a}^{38}$ reported that clove oil showed an MIC of $25 \mu \mathrm{L} / \mathrm{mL}$, which was lower. The conidial disc and fungal mycelium stages responded differently. The fungal mycelium disc growth was inhibited by fumigation with clove oil, whereas slightly less fungal mycelium inhibition was seen for cinnamon oil fumigation. This difference seems to result from cinnamon oil depressing conidial germination and conidial growth, while clove oil showed an acute effect in terms of fungal mycelium inhibition. For the fungal colony tests, volatile oil fumigation gave different result than the conidial disc test. Fumigation with clove oil presented excellent fungal inhibition compared with that for cinnamon oil. The oils also inhibited both atoxigenic and toxigenic fungal strains. This result indicated that the efficacy of essential oils depends on the fungal stage and application technique, such as fumigation and direct contact. Essential oils from various sources could have different antifungal effects for pathogen control. The concentrations of essential oils may affect fungi differently. This means that researchers should manage the active ingredient content inside essential oils. Some chemical components in essential oils are absent/ present depending on location, fertilization, harvesting site, and site weediness ${ }^{44,45}$.

The essential oil treatment resulted in abnormal characteristics in the A. flavus colony. The untreated fungal pathogen showed a green conidial head and high production of sclerotia. A $200 \mu \mathrm{L} / \mathrm{L}$ clove oil treatment of $A$. flavus affected the fungal colonies. The fungal vesicle was reduced in size, and the conidial head turned from dark green to slightly brown. At a higher clove concentration of $400 \mu \mathrm{L} / \mathrm{L}$, the conidial head and sclerotia were absent. The fungi showed only white mycelia on the culture medium. The increase in clove oil concentration showed a direct effect on fungal mycelium and sclerotium production. Similar activity on the fungal colony was observed with cinnamon treatment at $100 \mu \mathrm{L} / \mathrm{L}$. Total inhibition of colony growth was observed at 200 to $400 \mu \mathrm{L} / \mathrm{L}$. After observation of the abnormal character of treated $A$. flavus with a compound microscope, we found that the vesicle size was reduced. The conidiophores near the vesicle lost their integrity, and abnormal phialide were found, in contrast with the untreated fungi; similar to the results reported by Helal et $\mathrm{al}^{10,35}$, Carmo et $\mathrm{al}^{36}$, and Xing et $\mathrm{al}^{46}$. Fungi responded to essential oils by producing a thinner cell wall or smaller fungal mycelia, and the conidial head shrank and lacked conidiophores. Cinnamaldehyde, a cinnamon oil component, shrinks fungal hyphae and causes some depression of the hyphal surface ${ }^{37,47}$. Phuangsri et al $^{48}$ described that treating $A$. flavus with Zanthoxylum piperitum seed oil decreased the diameter of the hyphae and distorted the conidial head. Tian et al ${ }^{49,50}$ studied the effects of thymol and Cinnamomum jensenianum essential oil on morphological characteristics, finding such changes as decreased conidial head size, a lack of sporulation, and a disturbed plasma membrane, fibrillar layer, and cytoplasm. The antifungal essential oil properties of this oil disturb the functions related to ergosterol.

Our study found that the fungal cell wall ergosterol content in toxigenic $A$. flavus immediately decreased after treatment with essential oils. As ergosterol is a chemical component in the fungal cell membrane ${ }^{51}$, it plays a role in membrane fluidity, regulation, activity, and the cell membrane control of the cell cycle ${ }^{52,53}$. At some concentrations after treatment with an essential oil, the fungi showed a decrease in ergosterol content. Ahmad 
et $\mathrm{al}^{54}$ referred to eugenol as having an antifungal mechanism similar to fluconazole in treatments of Aspergillus sp. This mechanism may cause essential oil to block ergosterol synthesis, therefore reducing the ergosterol content in the fungal cell membrane ${ }^{55}$. Undoubtedly, we observed increasing electroconductivity in the clove and cinnamon oil-treated samples. The fungal cell wall structure change caused the leakage of fungal cytoplasm in the mycelia ${ }^{10,35}$. The thinner fungal mycelia caused $\mathrm{Ca}^{+2}, \mathrm{~K}^{+}$, and $\mathrm{Mg}^{2+}$ leakage from inside the fungal mycelia. These essential oils then affected the ergosterol content in the plasma membrane of the tested fungi ${ }^{56}$. This change will reduce aflatoxin accumulation by fungi.

\section{ACKNOWLEDGMENTS}

This work was supported by the Postharvest Technology Innovation Center, Ministry of Higher Education, Science, Research and Innovation, Bangkok, Thailand.

\section{CONFLICT OF INTEREST}

The authors declare that there is no conflict of interest.

\section{AUTHORS' CONTRIBUTION}

All authors listed have made a substantial, direct and intellectual contribution to the work, and approved it for publication.

\section{FUNDING}

This work was supported by the Postharvest Technology Innovation Center, Ministry of Higher Education, Science, Research and Innovation, Bangkok, Thailand. The project number is PBM.P.4/2560.

\section{DATA AVAILABILITY}

All datasets generated or analyzed during this study are included in the manuscript.

\section{ETHICS STATEMENT}

This article does not contain any studies with human or animals performed by any of the authors.

\section{REFERENCES}

1. Samson RA, Visagie CM, Houbraken J, et al. Phylogeny, identification and nomenclature of the genus
Aspergillus. Studies in Mycology. 2014;78:141-173. doi: 10.1016/j.simyco.2014.07.004

2. Frisvad JC. Taxonomy, chemodiversity, and chemoconsistency of Aspergillus, Penicillium, and Talaromyces species. Front Microbiol. 2015;5:773. doi: 10.3389/fmicb.2014.00773

3. Cotty PJ. Virulence and cultural characteristics of two Aspergillus flavus strains pathogenic on cotton. Phytopathology. 1989;79:808-814. doi: 10.1094/ phyto-79-808

4. Geiser DM, Dorner JW, Horn BW, Taylor JW. The phylogenetics of mycotoxin and sclerotium production in Aspergillus flavus and Aspergillus oryzae. Fungal Genet Biol. 2000;31(3):169-179. doi: 10.1006/ fgbi.2000.1215

5. Perrone G, Gallo A, Logrieco AF. Biodiversity of Aspergillus section Flavi in Europe in relation to the management of aflatoxin risk. Front Microbiol. 2014;5:377. doi: 10.3389/fmicb.2014.00377

6. Atehnkeng J, Donner M, Ojiambo PS, et al. Environmental distribution and genetic diversity of vegetative compatibility groups determine biocontrol strategies to mitigate aflatoxin contamination of maize by Aspergillus flavus. Microb Biotechnol. 2016;9(1):7588. doi: 10.1111/1751-7915.12324

7. Varga J, Baranyi N, Chandrasekaran M, Vagolgyi C, Kocsube S. Mycotoxin producers in the Aspergillus genus: an update. Acta Biol Szeged. 2015;59(2):151167. http://abs.bibl.u-szeged.hu/index.php/abs/ article/view/2878/2870. Accessed March 25, 2020.

8. Hell K, Mutegi C, Fandohan P. Aflatoxin control and prevention strategies in maize for Sub-Saharan Africa. Julius-Kuhn-Archiv. 2010;425:534-541. doi: 10.5073/ jka.2010.425.388.

9. Hell K, Cardwell KF, Setamou M, Poehling HM. The influence of storage practices on aflatoxin contamination in maize in four agroecological zones of Benin, West Africa. J Stored Prod Res. 2000;36(4):365382. doi: 10.1016/S0022-474X(99)00056-9

10. Helal GA, Sarhan MM, Abu Shahla ANK, Abou El-Khair EK. Effects of Cymbopogon citratus L. essential oil on the growth, morphogenesis and aflatoxin production of Aspergillus flavus ML2-strain. J Basic Microbiol. 2007;47(1):5-15. doi: 10.1002/jobm.200610137

11. Tian J, Ban $\mathrm{X}$, Zeng $\mathrm{H}$, Huang $\mathrm{B}, \mathrm{He} \mathrm{J}$, Wang $\mathrm{Y}$. In vitro and in vivo activity of essential oil from dill (Anethum graveolens L.) against fungal spoilage of cherry tomatoes. Food Control. 2011;22(12):1992-1999. doi: 10.1016/j.foodcont.2011.05.018

12. Shao X, Cheng S, Wang H, Yu D, Mungai C. The possible mechanism of antifungal action of tea tree oil on Botrytis cinerea. J Appl Microbiol. 2013;114(6):16421649. doi: 10.1111/jam.12193

13. Avanco GB, Ferreira FD, Bomfim NS, et al. Curcuma longa $\mathrm{L}$. essential oil composition, antioxidant effect, and effect on Fusarium verticillioides and fumonisin production. Food Control. 2017;73:806-813. doi: 10.1016/j.foodcont.2016.09.032

14. Hu Y, Zhang J, Kong W, Zhao G, Yang M. Mechanism of antifungal and anti-aflatoxigenic properties of essential oil derived from turmeric (Curcuma longa L.) on Aspergillus flavus. Food Chem. 2017;220:1-8. 
doi: 10.1016/j.foodchem.2016.09.179.

15. Huang Y, Zhao J, Zhou L, et al. Antifungal activity of the essential oil of Illicium verum fruit and its main component trans-Anethole. Molecules. 2010;15(11):7558-7569. doi: 10.3390/ molecules15117558

16. Lucas GC, Alves E, Pereira RB, Perina FJ, Souza RM. Antibacterial activity of essential oils on Xanthomonas vesicatoria and control of bacterial spot in tomato. Pesq Agropec Bras. 2012;47(3):351-359. doi: 10.1590/ S0100-204X2012000300006

17. Tenne PCRK, Karunaratne MMSC. Phytochemical profile and bioactivity of essential oil from Pimenta dioica leaves on cowpea beetle, Callosobruchus maculatus (F.) (Coleoptera: Bruchidae): A farmer friendly solution for postharvest pest management. Open Agric. 2018;3(1):301-309. doi: 10.1515/opag2018-0033

18. Zaka SM, Iqbal N, Saeed Q, et al. Toxic effects of some insecticides, herbicides, and plant essential oils against Tribolium confusum Jacquelin du val (Insecta: Coleoptera: Tenebrionidae). Saudi J Biol Sci. 2019;26(7):1767-1771. doi: 10.1016/j. sjbs.2018.05.012

19. Bluma RV, Etcheverry MG. Application of essential oils in maize grain: impact on Aspergillus section Flavi growth parameters and aflatoxin accumulation. Food Microbiol. 2008;25(2):324-334. doi: 10.1016/j. fm.2007.10.004

20. Chen C, Cai N, Chen J, Wan C. Clove essential oil as an alternative approach to control postharvest blue mold caused by Penicillium italicum in citrus fruit. Biomolecules. 2019;9:197. doi: 10.3390/biom9050197

21. Raper BK, Austwick PKC, Fennell DI. The genus Aspergillus. Robert E. Krieger Publishing Co., Inc., New york. 1977.

22. Yamamoto-Ribeito GMM, Grespan R, Kohiyama CY, et al. Effect of Zingiber officinale essential oil on Fusarium verticillioides and fumonisin production. Food Chemistry. 2013;141(3):3147-3152. doi: 10.1016/j. foodchem.2013.05.144

23. Ferreira MF, Hirooka EY, Ferreira F, Silva MV, Mossini SAG, Machinski Jr M. Effect of Zingiber officinale Roscoe essential oil in fungus control and deoxynivalenol production of Fusarium graminearum Schwabe in vitro. Food Addit Contam Part A. 2018;35(11), 21682174. doi: $10.1080 / 19440049.2018 .1520397$

24. White TJ, Bruns T, Lee S, Taylor J. Amplification and direct sequencing of fungal ribosomal RNA genes for phylogenetics. PCR Protocols: A guild to method and application, Academic Press, Massachusetts. 1990. doi: 10.1016/B978-0-12-372180-8.50042-1

25. Irinyi L, Serena C, Garcia-Hermoso D, et al. International Society of Human and Animal Mycology (ISHAM)ITS reference DNA barcoding database-the quality controlled standard tool for routine identification of human and animal pathogenic fungi. Med Mycol. 2015;53(4):313-337. doi: 10.1093/mmy/myv008

26. Riaz A, Rasib KZ, Aslam H, Raza S. Molecular identification of Aspergillus flavus using inter transcribed spacers (ITS). Biotechnol J Int. 2017;20(4):18. doi: 10.9734/BJI/2017/33951
27. Cotty PJ. Aflatoxin-producing potential of communities of Aspergillus section Flavi from cotton producing areas in the United States. Mycol Res. 1997;101(6):698-704. doi: $10.1017 /$ S0953756296003139

28. Saito $M$, Tsuruta $O$. A new variety of Aspergillus flavus from tropical soil in Thailand and its aflatoxin productivity. JSM Mycotoxin. 1993;37:31-36. doi: 10.2520/myco1975.1993.31

29. Benito N, Carranza C, Magnoli C, Barberis C. Survey and aflatoxingenic for Aspergillus section Flavi from three production regions of Argentina. Cienc. Suelo (Argentina). 2018;36(2):82-91. https:// ri.conicet.gov.ar/bitstream/handle/11336/105186/ CONICET_Digital_Nro.8efd6a0c-510f-4b79-ba39cec8283c7922_A.pdf? sequence $=2 \&$ isAllowed $=y$. Accessed March 25, 2020.

30. Goto T, Wicklow DT, Ito Y. Aflatoxin and cyclopiazonic acid production by a sclerotiumproducing Aspergillus tamarii strain. Appl Environ Microbiol. 1996;62(11):4036-4038. doi: 10.1128/ aem.62.11.4036-4038.1996

31. Rattanakreetakul C, Rangsuwan S, R. Pongpisutta, P. Keawmanee. Differentiation on secondary metabolites of toxigenic and atoxigenic Aspergillus flavus. Khon Kaen Agr J. 2020;48:693-702. doi: 10.14456/kaj.2020.64

32. Mamo FT, Shang B, Selvaraj JN, Wang Y, Liu Y. Isolation and characterization of Aspergillus flavus strains in China. J Microbiol. 2018;56(2):119-127. doi: 10.1007/ s12275-018-7144-1

33. Maruzzella JC, Sicurella NA. Antibacterial activity of essential oil vapors. J Am Pharm Assoc. 1960;49(11):692-694. doi: 10.1002/jps.3030491103

34. Maruzzella JC, Chiaramonte JS, Garofalo MM. Effects of vapors of aromatic chemicals on fungi. J Pharm Sci. 1961;50(8):665-668. doi: 10.1002/jps.2600500809

35. Helal GA, Sarhan MM, Abu Shahla ANK, Abou EIKhair EK. Effects of Cymbopogon citratus L. essential oil on the growth, lipid content and morphogensis of Aspergillus niger ML2-strain. J Basic Microbiol. 2006;46(6):456-469. doi: 10.1002/jobm.200510106

36. Carmo ES, Lima EO, De Souza EL, De Sousa F. Effect of Cinnamomum zeylanicum blume essential oil on the growth and morphogenesis of some potential pathogenic Aspergillus species. Braz J Microbiol. 2008;39(1):91-97. doi: 10.1590/s1517838220080001000021

37. Sun Q, Shang B, Wang L, Lu Z, Liu Y. Cinnamaldehyde inhibits fungal growth and aflatoxin $B 1$ biosynthesis by modulating the oxidative stress response of Aspergillus flavus. Appl Microbiol Biotechnol. 2016;100(3):13551364. doi: 10.1007/s00253-015-7159-z

38. Xing Y, Xu Q, Li X, Che Z, Yun J. Antifungal activities of clove oil against Rhizopus nigricans, Aspergillus flavus and Penicillium citrinum in vitro and in wounded fruit test. J Food Saf. 2012;32(1):84-93. doi: 10.1111/j.17454565.2011.00347.x

39. Hua $H$, Xing F, Selvaraj JN, et al. Inhibitory effect of essential oils on Aspergillus ochraceus growth and ochratoxin A production. PLoS One. 2014;9(9):e108285. doi: 10.1371/journal.pone.0108285

40. Li Y, Kong W, Li M, et al. Litsea cubeba essential oil as 
the potential natural fumigant: Inhibition of Aspergillus flavus and AFB1 production in licorice. Ind Crops Prod. 2016;80:186-193. doi: 10.1016/j.indcrop.2015.11.008

41. Rangsuwan S, Rattanakreetakul C, Pongpisutta R, Keawmanee P. Post-Harvest control of anthracnose disease on mango and chili with cinnamon oil and extracts of medicinal plants mixture from natural products. Agri Sci J. 2017;48(3)(Suppl.):93-96. https:// www.phtnet.org/download/phtic-seminar/1715.pdf. Accessed March 25, 2020.

42. Rattanakreetakul C, Fauzi AA, Keawmanee P, Rangsuwan S. Stem-end rot disease retardation in banana fruit with clove oil fumigation under incubator. Agri Sci J. 2017;48(3)(Suppl.):105-108. https://www. phtnet.org/download/phtic-seminar/1718.pdf. Accessed March 25, 2020.

43. Gong A, Wu N, Kong X, et al. Inhibitory effect of volatiles emitted from Alcaligenes faecalis N1-4 on Aspergillus flavus and aflatoxins in storage. Front Microbiol. 2019;10:1419. doi: 10.3389/fmicb.2019.01419

44. Gil A, Fuente EB, Lenardis AE, et al. Coriander essential oil composition from two genotypes grown in different environmental conditions. J Agric Food Chem. 2002;50(10):2870-2877. doi: 10.1021/jf011128i

45. Noudjou F, Kouninki $H$, Ngamo LST, et al. Effect of site location and collecting period on the chemical composition of Hyptis spicigera Lam. an insecticidal essential oil from north-Cameroon. J Essent Oil Res. 2007;19(6):597-601. doi: 10.1080/10412905.2007.9699340

46. Xing F, Hua H, Selvaraj JN, et al. Growth inhibition and morphological alterations of Fusarium verticillioides by cinnamon oil and cinnamaldehyde. Food Control. 2014;46:343-350. doi: 10.1016/j. foodcont.2014.04.037

47. Brodowska KM, Brodowska AJ, Smigielski K, LodygaChruscinska E. Antioxidant profile of essential oils and extracts of cinnamon bark (Cinnamomum cassia). Eur J Biol Res. 2016;6(4):310-316. doi: 10.5281/ zenodo.197200

48. Phuangsri C, Nuntawong N, Niamsup H. Antifungal activity of the essential oil extracted from Zanthoxylum piperitum seeds against Aspergillus flavus. Chiang Mai J Sci. 2017;44(2):584-594. http://cmuir.cmu.ac.th/ jspui/handle/6653943832/56774. Accessed March 25, 2020.

49. Tian J, Huang B, Luo X, et al. The control of Aspergillus flavus with Cinnamomum jensenianum Hand.Mazz essential oil and its potential use as a food preservative. Food Chem. 2012;130(3):520-527. doi: 10.1016/j.foodchem.2011.07.061

50. Tian F, Woo SY, Lee SY, Chun HS. p-Cymene and its derivatives exhibit antiaflatoxigenic activities against Aspergillus flavus through multiple modes of action. Appl Biol Chem. 2018;61:489-497. doi: 10.1007/ s13765-018-0382-4

51. Alcazar-Fuoli L, Mellado E. Ergosterol biosynthesis in Aspergillus fumigatus: its relevance as an antifungal target and role in antifungal drug resistance. Front Microbiol. 2013;3:439. doi: 10.3389/fmicb.2012.00439

52. Bard M, Lee ND, Turi T, et al. Sterol synthesis and viability of erg11 (cytochrome P450 I anosteroldemethylase) mutations in Saccharomyces cerevisiae and Candida albicans. Lipids. 1993;28(11): 963-967. doi: 10.1007/ bf02537115

53. Gooday GW. The dynamics of hyphal growth. Mycol Res. 1995;99(4):385-394. doi: 10.1016/S09537562(09)80634-5

54. Ahmad A, Khan A, Manzoor N, Khan LA. Evolution of ergosterol biosynthesis inhibitors as fungicidal against Candida. Microb Pathog. 2010;48(1):35-41. doi: 10.1016/j.micpath.2009.10.001

55. Bendaha $\mathrm{H}, \mathrm{Yu} \mathrm{L}$, Touzani R, et al. New azole antifungal agents with novel modes of action: Synthesis and biological studies of new tridentate ligands based on pyrazole and triazole. Eur J Med Chem. 2011;46(9):4117-4124. doi: 10.1016/j. ejmech.2011.06.012

56. Venkatesan R, Karuppiah PS, Arumugam G, Balamuthu K. $\beta$-Asarone exhibits antifungal activity by inhibiting ergosterol biosynthesis in Aspergillus niger ATCC 16888. Proc Natl Acad Sci, India, Sect. B Biol Sci. 2019;89:173-184. doi: 10.1007/s40011-017-0930-4 